\title{
RECURRENCE OF NOVA EXPLOSIONS
}

\author{
Kyoji Nariai \\ Tokyo Astronomical Observatory, University of Tokyo
}

and

Ken'ichi Nomoto

Department of Physics, Ibaraki University

It has been shown by many computations that a nova explosion is triggered by mass accretion onto a white dwarf in a close binary system. such nova explosions will recur many times in the following way. When a certain amount of hydrogen-rich gas has been accreted on the white dwarf, a hydrogen-shell flash is ignited. Then the hydrogen-rich envelope expands greatly, which, in some cases, grows into a nova explosion. Almost all envelope mass is ejected into space or at least overflows its Roche lobe. After that the mass of the envelope increases again by accretion and the shell flash is ignited again. The period of such recurrence is given by $\tau_{\text {rec }}=\Delta \mathrm{M}_{\mathrm{H}} /(\mathrm{dM} / \mathrm{dt})$, where $\mathrm{dM} / \mathrm{dt}$ and $\Delta \mathrm{M}_{\mathrm{H}}$ are the rate of accretion and the mass contained in the hydrogen-rich envelope at the ignition point.

We have calculated such recurrence period $\tau_{\text {rec }}$ as follows. During the many cycles of the shell flashes, the thermal structure of the accreted envelope approaches a thermally steady state. In this state, the entropy distribution $s(q, t)$ is steady in the reference frame of mass fraction $q \equiv M_{r} / M, i . e$. , addition of entropy due to mass accretion is just balanced with radiative heat transport. Such a steady state does not depend on the initial luminosity of the white dwarf but is determined only by the mass of the white dwarf $M$, and $d M / d t$. For the set of parameters $(M, d M / d t)$, we have computed the steady state structure exactly and obtained the value of $\Delta \mathrm{M}_{\mathrm{H}}$ at the ignition point. The results are shown in Fig. 1 , where the relations between $\mathrm{dM} / \mathrm{dt}$ and $\Delta \mathrm{M}_{\mathrm{H}}$ or $\tau_{\text {rec }}$ are given. It is found that the observed recurrent. novae having $\tau_{\text {rec }}$ of several tens of years take place only for massive white dwarfs of $M \geqslant 1.2 M_{\odot}$ with relatively rapid accretion.

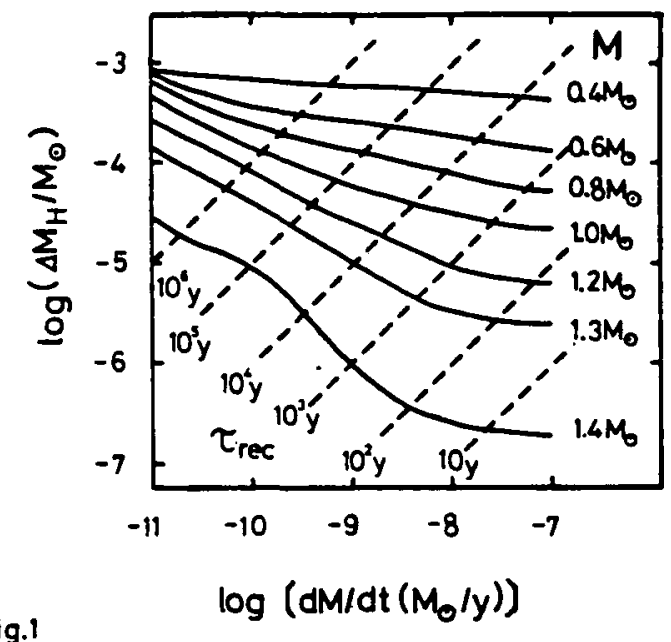

